La puesta en escena de un espectáculo teatral es un proceso creativo complejo en el que intervienen múltiples agentes, elementos y procesos de significación. El texto deviene pretexto y por eso la obra literaria dará lugar a una serie de textos sucesivos que se concretarán en el texto teatral, aquel que se actualiza en cada representación. En ese proceso cobra especial relevancia la figura del dramaturgista, pero también la del traductor, pues si la dramaturgia implica revisión y/o adecuación de la materia literaria a la sintaxis escénica, la traducción facilita el acceso a textualidades de otras lenguas. Con este artículo queremos proponer un marco teórico desde el que estudiar la relación entre teatro y traducción a partir del análisis de los conceptos, procesos y métodos de trabajo que son específicos de ese territorio de intersección de prácticas culturales.

Palabras Clave: texto dramático, dramaturgia, escenificación, puesta en escena, teatro, traducción.

\title{
Teatro y traducción / Por un giro escénico
}

\author{
MANUel F. VIEITES \\ Escuela Superior de Arte Dramático de \\ Galicia
}

Universidad de Vigo, grupo BITRAGA

\section{Theatre and translation. Towards an scenic turn}

The enactment of a theatrical performance is a complex creative process that involves multiple agents, elements and processes of signification. The text becomes a pretext and so the literary work results in a series of successive texts that will materialize in the theatrical text, that which is heard and seen during each one of the performances. The figure of the dramaturge is particularly important in the overall process, but so it is that of the translator, due to the fact that if drama involves the revision and/or adaptation of the literary material to the scenic syntax, translation facilitates the access to texts written in other languages. With this article we propose a tentative theoretical framework in order to consider the relationship between theatre and translation based on the analysis of the concepts, processes and working methods that are specific to this field of intersection of cultural practices.

KEYWORDS: drama, dramaturgy, staging, production, theatre, translation. 


\section{INTRODUCCIÓN}

Hace ya algunos años, en la sede de la Asociación de Directores de Escena de España se

112 recibía una carta de la Sección Autónoma de Traductores de Libros, de la Asociación Colegial de Escritores de España (ACE Traductores), en la que la agrupación profesional planteaba la necesidad de entablar conversaciones para delimitar territorios, establecer relaciones de buena vecindad y cooperar, en lo posible, en el respeto y defensa del trabajo y de los derechos intelectuales de unos y otros. Se trataría de determinar, en suma, una especie de ámbito común de intereses, debido, en parte, a la existencia de aquello que Melendres (1999: 40), hablando de la interacción de los oficios todos del teatro, definía como suma de «derechos artísticos igualmente legítimos y defendibles» y que situaba en la intersección entre la propiedad horizontal y la propiedad vertical, símil muy acertado si pensamos en lo que se viene denominando edificio teatral. Reconociendo la oportunidad y la necesidad de ese diálogo, poco después la Revista ADE/Teatro, que edita la citada Asociación de Directores, publicaba en su número 153 la transcripción de una mesa de trabajo en la que personas vinculadas a la traducción y a la dirección escénica debatían en torno al tema propuesto bajo el lema: «El respeto para los traductores, un problema latente en el teatro español» (VV. AA., 20I4: I4-33). En esa mesa de debate, Miguel Sáenz, traductor y miembro de número de la Real Academia Española, decía (VV. AA., 20I4: I7):

La mejor solución es cuando el traductor está trabajando con los actores mientras se está montando la obra porque cualquier cambio se puede discutir. Pero rara vez ocurre que el traductor tenga el control de sus traducciones. Lo que hace es ceder el uso de su traducción con la reserva de que si hay cambios, que le consulten. La situación este año ha sido especialmente grave, porque parece que todo

En ese mismo número se publicaba, como complemento a la mesa de debate, un «Código de buenas prácticas para traducciones y adaptaciones teatrales» (ACE/ADE, 20I4: 33), pues no estamos ante un problema menor, ni en España ni en otros países en los que la traducción ni se ha legitimado ni se ha institucionalizado como ámbito profesional especializado, y menos en un sector como el de la traducción para el campo teatral, en el que los caminos por los que el texto llega a la escena son múltiples y a veces demasiado oscuros y tortuosos.

Como traductor de textos dramáticos y de ensayo teatral, pero sobre todo como profesor en una Escuela Superior de Arte Dramático (en la que se forman creadores escénicos en los ámbitos de la interpretación, la dirección escénica, la dramaturgia o la escenografía), entiendo que estamos ante una cuestión especialmente relevante y que no obstante no ha tenido especial eco en estudios específicos o en revistas especializadas, más centradas en el análisis de casos (Arqués, 2015), en el desarrollo de estrategias y metodologías de trabajo aplicables en procesos generales (Zatlin, 2005) o específicos (Summers, 20I6), o en interesantes debates teóricos (Aaltonen, 2000; Buzelin, 2005; Ghignoli, 2013). Sin embargo, la cuestión es transcendental por cuanto no sólo afecta al ejercicio profesional en la traducción, y a las posibilidades de su desarrollo, sino también a los derechos que el traductor tiene sobre su obra traducida (y la que deja de traducir debido al intrusismo creciente) y sobre todo aquello que esa obra acabe siendo. Atendiendo a esas derivas imprevistas del texto, 
en las que hay aspectos éticos y deontológicos, la cuestión también afecta a la formación de todas aquellas personas que en un momento determinado de su vida profesional puedan precisar de un texto traducido o encontrarse con uno en un proceso de creación escénica.

Por eso, siguiendo a Melendres, es necesario analizar procesos, propiedades y derechos, y en este trabajo queremos ofrecer una visión general de ese amplio territorio que tradicionalmente se quiere abarcar con el sintagma teatro y traducción (Ezpeleta, 2007, 2009), intentando establecer con precisión los agentes que habitan el territorio, sus funciones, los textos que generan y las finalidades de los mismos. Definir conceptos y territorios nos ayuda a establecer con precisión las funciones y las responsabilidades de unos y de otros para mejor articular una convivencia siempre necesaria, por el bien del teatro y de la cultura, pero también para anticipar y proponer debates necesarios en torno a las especificidades de la traductología teatral (Espasa, 2009; Marinetti, 2013; Lapeña, 2014), y situarlos en el ámbito de la escena, allí donde el teatro se genera. Entendemos que considerar el tránsito que lleva de un texto fuente a un espectáculo presentado en escena, ayuda a comprender el rol del traductor en los diferentes procesos, y como afirmaba Curell (2013) no sólo nos permite realizar un análisis sistemático del hecho de la traducción, en este caso teatral, sino también considerar sus implicaciones didácticas en la formación del traductor, pero igualmente en la educación de los creadores escénicos. Por ello, delimitar espacios posibles de encuentro entre traducción y creación teatral debiera ayudarnos a poner en valor el rol que la traducción especializada y realizada por especialistas puede aportar en la mejora y en la excelencia del arte teatral.

Lo hacemos en una perspectiva escénica, entendiendo que nuestra visión del problema puede complementar otras lecturas del mismo, como las que proponen Zuber (I980), Aaltonen (2000), o Bassnett (2002), pero además señalando que es difícil concordar con afirmaciones 113 como la de esta última autora al decir que en un texto teatral leemos «something incomplete, rather than as a fully rounded unit» (2002: II9), pues defendemos con Veltrusky que el texto dramático es un producto cultural de naturaleza literaria en el que el lenguaje, salvo excepciones, «constituye el único material» (I990: 15), en tanto el teatro utiliza otros elementos de significación (Barthes, I967; Kowzan, I969), otros procesos de construcción de significantes y significados, y otros modos de comunicación (Vieites, 20I6a). Por eso el texto dramático, contrariamente a esa visión platónica del texto (Bobes Naves, I987) está completo en tanto obra literaria, en su literariedad y en su dramaticidad.

Como complemento de otros "giros" (Snell-Hornby, 2006), como puedan ser el «sociológico» (Buzelin, 2005), el «semiótico» (Pavis I992), el «sociocultural» o «cultural» (Scolnicov, Holland et al., I989; Bassnett, 2002, 20I2; Zhang, 2013), o incluso el "performativo» (Aaltonen, 2013), ${ }^{\mathrm{I}}$ en el fondo complementarios entre sí, y sin olvidar lo que aportan las teorías de la recepción en su aplicabilidad al campo

El concepto de «performance», utilizado por Chomsky para indicar una «acción» o «actuación» en el ámbito del desempeño expresivo, toma en el campo de las artes escénicas sentidos diferentes, sea para referir una presentación escénica (Carlson, 2003) sea para indicar un modo de actuación que ya fue intuido por Evreinov (1927) y que desarrollan Burke (1945) o Goffman (1959, 1974), pero también Schechner (2000). En traductología se ha aplicado en no pocas ocasiones a la idea de «representabilidad» (Espasa, 2009), equiparando dramaticidad y teatralidad, y olvidando que tales conceptos remiten a dos procesos de contrucción de significantes y significados diferentes que operan en modo diferente en los niveles morfológico, sintáctio, semántico y pragmático; el primero con palabras y el segundo con el «espesor de signos» del que hablaba Barthes (1967: 310). 
(Nord, 2005), lo que proponemos es un «giro escénico» para considerar la traducción del texto dramático desde la escena y en función de los procesos que ese texto pueda general al situarse en el contexto de una escenificación que genera una puesta en escena y un espectáculo (Martínez Paramio, 2006). Entendemos además que el «giro» propuesto es el que mejor se aviene a la naturaleza del problema que motiva el presente trabajo, ${ }^{2}$ y en especial para hacer nuestras las palabras e intenciones de Aaltonen (2013: 386), para visibilizar lo que define como «invisible actors», no solo en la perspectiva de la traducción sino también en la de la escenificación, y en la recepción del conjunto.

\section{LAS VOCES DEL TEXTO Y SUS FUNCIONES}

Como señalaba el profesor García Barrientos (I991: 22) sigue siendo habitual que cualquier estudio que contenga en su título la palabra teatro comience por una definición, que a veces ha de ser demorada, del término, para diferenciar con claridad lo que se construye con palabras, $y$ por tanto es literatura, y aquello que configura lo que Barthes definió como máquina cibernética y que genera un espesor de signos (1967: 3093ro). Por fortuna, aumenta día a día la visión del teatro como un arte complejo en el que el texto dramático es un elemento de significación, de mayor o menor importancia, que, junto a otros, permite configurar el hecho central del arte dramático: la comunicación directa entre un actor $y$ un espectador (Grotowski, I970: I3).

2 Cuando consideramos propuestas como las que edi$\tan$ Bassnett y Lefevere (1998) en torno a la traducción como manipulación y reescritura, no podemos dejar de recordar las prácticas traductológicas que se desarrollaban en España en el siglo xviII, en especial las vinculadas con el texto dramático, que en muchos casos apostaban por similares estrategias, o el hecho de que Margarita Hickey y Pellizzoni en el «Prólogo» a su traducción de la Andrómaca de Racine demandaba un mayor respeto por el original (I789: VII-VIII).
Tradicionalmente, desde el ámbito de los estudios literarios se ha entendido el proceso de realización de un espectáculo como una traducción (o suma de traducciones) orientada a ilustrar un texto a partir de la conversión de signos de carácter verbal en signos de carácter oral, visual, cinético, corporal, gestual, olfativo... (Bobes Naves, 1987). Desde esa perspectiva, más dominante de lo que cabría creer, el rol del equipo de realización teatral (actores, actrices, directoras, técnicos, diseñadoras...) en tanto traductores o conversores de signos, no sería otro que seguir el hilo del texto dramático, en un proceso en el que incluso cabría hablar de transducción (Doležel, 1999). ${ }^{3}$ En esa visión también encontramos una de las razones de que se valorase tan positivamente la fidelidad escénica al texto traducido como signo de reverencia al texto fuente, en la medida en que desde esa perspectiva lo realmente importante de un espectáculo teatral sería su componente verbal, sus palabras, para mantener el espíritu y la letra del autor, de su texto. Por eso era habitual, y todavía lo es, que alguien acabe preguntando: «¿En cuántos bytes se desvía cada función del texto del libreto?» (Fernández Cardo, I995: 406). Pero ante esa visión logocéntrica, también hay voces que proponen considerar el espectáculo teatral como un artefacto cultural con rasgos diferenciales, en el que el texto no deja de ser un pretexto más, con lo que su lectura no es unívoca. Como explicaba Mateo (1995: 99-100):

3 Esa centralidad de la palabra, y del texto en su «literariedad», fue el motivo de que durante tantos y tantos años se afirmase, con una contundencia abrumadora, que algunos autores eran irrepresentables (Valle-Inclán puede ser un buen ejemplo), partiendo de una lectura platónica de la creación escénica que explicaba que el espectáculo no era más que una lectura incompleta (acto) de un texto (potencia, suma de virtualidades). Sobre esa visión platónica se asientan algunas propuestas y/o debates sobre la «representabilidad», pues parten de la una visión subsidiaria del espectáculo frente al texto (Espasa, 2009). 
While there is only one source text, there is no limit to the number of target texts that can be drawn from it, as each culture appropriates a foreign text differently. Similarly, one single drama text may be performed in many different ways, since each production realizes what each producer can or wants to read and see in the text.

En efecto, es importante destacar que texto dramático y espectáculo teatral, aun cuando mantengan una evidente relación como artefactos culturales, son objetos distintos, en su morfología, en su sintaxis, en su semántica y en su pragmática. Veltrusky lo expresaba con precisión al decir que el teatro "no constituye otro género literario sino otro arte» (I990: 15). Pero que sea necesario distinguir literatura y teatro como dos productos culturales diferenciados en los niveles morfológico, sintáctico, semántico y pragmático, no invalida el hecho de que uno de los pretextos habituales en la creación escénica es el texto dramático, y en los sistemas teatrales normalizados el número de textos traducidos es sumamente elevado. Si en ese tránsito que va de la intención previa al espectáculo (Fábregas, I975) se parte en muchos casos del texto dramático como una de las materias primas de aquel, resulta fundamental la consideración del roly de las funciones del traductor.

Pero para considerar el rol de la traducción y de la dramaturgia, como disciplinas que operan sobre el texto, una de las primeras cuestiones al abordar una obra dramática y en la perspectiva inicial de la lectura, consiste en considerar las voces presentes en ella, pues estamos ante un artefacto literario complejo en el que se entrelazan diferentes tipos de textos con funciones y finalidades diferentes. Siguiendo aportaciones de Tacca (I985) o Villegas (I99I), entendemos que en el texto dramático cabe considerar hasta cinco niveles de escritura y concreción, que se muestran en la tabla $\mathrm{I}$.

TABLA I: LAS VOCES DEL TEXTO DRAMÁTICO. ElabORACIÓN PROPIA

\begin{tabular}{lll}
\hline TIPO & MANIFESTACIÓN & EMISOR \\
\hline PARATEXTO $^{4}$ & $\begin{array}{l}\text { Imágenes, tipografías, textos en contra- } \\
\text { portada, textos en solapa interior. }\end{array}$ & Editor. \\
\hline PERITEXTO & Título, prólogos, epílogos, dedicatorias. & Dramaturgo empírico. \\
\hline $\begin{array}{l}\text { TEXTO DRAMÁTICO } \\
\text { PRIMARIO }\end{array}$ & Diálogos. & Personajes. \\
\hline $\begin{array}{l}\text { TEXTO DRAMÁTICO } \\
\text { SECUNDARIO }\end{array}$ & $\begin{array}{l}\text { Didascalias explícitas (funcionales y } \\
\text { expresivas). }\end{array}$ & Dramaturgo implícito. \\
\hline $\begin{array}{l}\text { TEXTO DRAMÁtico } \\
\text { ESPECTACULAR }\end{array}$ & Didascalias espectaculares. & Dramaturgista ficticio 5 \\
\hline
\end{tabular}

4 Siguiendo a Genette (1987), nos situamos en el territorio de lo que se ha denominado "paratraducción», lo que implica trasladar textos, imágenes y otros elementos que en ocasiones no son originales del autor del texto dramático, cuanto del editor o de otras voces (Garrido Vilariño, 2004; Yuste Frías, 20Io).

5 Como refiere García May en la presentación del volumen La palabra que empieza por D (Luckhurst, 2008), el término, tomado del alemán «dramaturg», como se dirá, presentó en su día una considerable complejidad y provocó una revolución intelectual y conceptual, especialmente en países de expresión inglesa o castellana. En torno al concepto, véase Pérez-Rasilla (2008) o Vieites (20I6c). 
Veamos a continuación un ejemplo de los tres últimos niveles de producción textual sacados de una obra de Domingo Miras, Las alum-
(...) Los cuatro, con sendos incensarios, lanzan olorosas nubes hacia los negros paramentos, envolviéndolos en una niebla gris, al tiempo que cantan.

coro.- (Mientras inciensan. Pueden oirse más voces dentro, si se desea.)

Per singulos dies

benedicimus te.

Et laudamus nomen tuum

in saeculum, et in saeculum secculi.

Renunciamos a una exposición más detallada en torno a estos niveles de creación textual y a su finalidad (Vieites, 2008), pues para los objetivos de este trabajo basta con señalar las diferencias existentes entre la voz que nos informa sobre el curso de la acción, y del tiempo y el espacio en el que se desarrolla (en cursiva), y la voz que sugiere posibilidades para un espectáculo posible (cursiva y subrayado), como también son diferentes los destinatarios a los que esas voces hablan. Mientras que el dramaturgo implícito, aquel que vive en la historia y la conoce, elabora el mundo dramático y da voz a los personajes que con él lo enhebran, pensando en el lector implícito, el dramaturgista ficticio, cuando emerge, propone ideas para un espectáculo ideal a un director igualmente implícito.

En ocasiones, estas dos últimas voces son especialmente relevantes. En el primer caso, la del dramaturgo implícito lo es en Act Without Words I, Act Without Words II o Krapp's Last Tape, tres obras de Samuel Beckett en las que la acción de los personajes se establece de forma $\tan$ milimétrica en el texto secundario que el más mínimo error en su escenificación (y en su traducción) puede desbaratar el juego escénico construido con palabras muy precisas. Para el segundo caso, la del dramaturgista ficticio, y siguiendo con el mismo autor, podemos considerar Play, en el que los focos juegan un papel especialmente relevante: «Spots off. Blackout. Five seconds. Strong spots simultaneously on three faces. Three seconds. Voices normal strength» (Beckett, 1990: 308). Para el caso en el que la voz de los personajes adquiere mayor relevancia, el propio Beckett nos ofrece un magnífico ejemplo en Not $I$, aquella obra en la que en un escenario con oscuro total una boca iluminada emite un discurso entrecortado.

Cada una de las voces señaladas cumple en cada texto una función específica, y en la traducción no cabe ser ajenos a esa función en tanto un texto dramático es mucho más que una suma de caracteres o palabras, pues el texto en definitiva configura un mundo que en sí mismo tiene una lógica y una coherencia propias, la que toda traducción debe salvaguardar, también para facilitar el contraste en el diálogo intercultural.

Porque si las voces del texto son importantes en tanto generan discursos diferentes, en ningún caso podemos perder de vista, en la traducción y en la puesta en escena, aquella importante propuesta de Hjelmslev en la que reclamaba «conceder gran importancia a la doble distinción entre forma y substancia y entre contenido (significado) y expresión (significante)", que le lleva a hablar de la forma y la substancia de la expresión y de la forma y la substancia del contenido ([1954] 1972: 47). Una distinción particularmente relevante que se deja sentir especialmente en la textualidad de autores dramáticos como Harold Pinter, en cuyas obras los aspectos formales y estilísticos tienen una clara dimensión semántica y pragmática, que afecta de forma clara a la morfología y a la sintaxis del espectáculo. Por eso la traducción de textos (dramáticos) para el teatro se torna una tarea sumamente compleja, que infelizmente se suele 
resolver, desde el campo de la creación teatral, prestando exclusiva atención a la substancia del contenido, a lo que tradicionalmente se denomina mensaje, olvidando que la forma y la substancia de la expresión también son mensaje, y una parte muy substantiva del mismo. ${ }^{6}$

Muchas veces los traductores olvidamos que en realidad al abordar un texto dramático tratamos con procesos de comunicación (Nord, 2005: 14), y por tanto con actos de habla. Pinter es un autor que suele prestar especial atención a la forma y a la substancia de la expresión para mostrar la naturaleza de las conductas que en un momento determinado activan y muestran las personas, sus actos de habla. Un buen ejemplo lo tenemos en The Room (Pinter, I996: Io8-Io9):

RILEY. Come home, Sal.

Pause.

Rose. What did you call me?

RILEY. Come home, Sal.

ROSE. Don't call me that.

RILEY. Come, now.

Rose. Don't call me that.

RILEY. So now you're here.

Rose. Not Sal.

RILEY. Now I touch you.

Rose. Don't touch me.

RILEY. Sal.

ROSE. I can't.

RILEY. I want you to come home.

ROSE. No.

RILEY. With me.

ROSE. I can't.

RILEY. I waited to see you.

ROSE. Yes.

6 Así ocurre, por ejemplo en la obra dramática de John Millington Synge, en la que sus personajes hablán en angloirlandés, una variedad diatópica del inglés con notables influencias del gaélico irlandes, de carácter fonético, morfológico y sintáctico, que configuran una forma específica de habla que caracteriza de forma precisa a los personajes. A modo de ejemplo tenemos obras como In the Shadow of the Glen (1903) o Deirdre of the sorrows (1909). Similares problemas plantean algunos textos de Martin $\mathrm{McD}$ onagh o de Marina Carr.
Estamos ante un diálogo de una fuerza y de una dureza extremas, por lo que el traductor ha de medir con exactitud las palabras que permiten construir la conducta de cada personaje, la situación comunicativa en la que la dicción, la prosodia y las formas de emisión juegan un papel importante (Escandell Vidal, 2005). Y por eso autores como Pinter o Beckett son tan difíciles, sea en la traducción, sea en la puesta en escena. Es importante no perder de vista esta cuestión, porque a la hora de hacer frente a la traducción de un texto dramático no estamos trabajando solo con palabras, sino con procesos de comunicación en los que los actos de habla tienen especial relevancia (Johnston, 20I3). Y es que el traductor no traduce un diálogo sino un proceso de comunicación, como bien explicaba Austin en el título de su obra más famosa, How to Do Things With Words (1962), y por ello la lectura demorada del texto es tan importante, para captar en toda su complejidad la dinámica relacional y comunicativa del mismo y su dimensión pragmática (Watzlawick, Bavelas y Jackson, I98I: I4I-I72).

\section{EL TEXTO, SUS LECTORES, LA TRADUCCIÓN Y LA DRAMATURGIA}

Si considerando las voces del texto llegamos a los niveles de concreción señalados, que suponen un trabajo diferenciado en el nivel de la traducción por cuanto se manifiestan habitualmente con registros diferentes, la lectura del texto, en tanto artefacto cultural de naturaleza normalmente lingüística que torna pertinentes los cuatro niveles señalados por Hjelmslev, presenta diferentes opciones que también configuran tipologías de lectura y lector:

- En un primer nivel, el de la lectura autotélica, podríamos hablar de un lector primario, para quien el texto dramático es básicamente un 
producto literario más, fuente de placer y de conocimiento.

- En un segundo nivel el texto puede ser objeto de una lectura especializada, sea por parte de la teoría y la historia literaria o por investigadores diversos de muy diferentes campos de conocimiento, y da lugar a un lector que acude al texto en busca de algo más que el simple placer de la lectura. Hablamos de un texto que puede ser leído, analizado e interpretado en función de los intereses que sobre él podría formular una historiadora, una socióloga, un lingüista, una psiquiatra, un profesor de literatura o una antropóloga, intereses que se definen en el nivel de su textualidad y de su literariedad, como un artefacto artístico y cultural dotado de referencialidad externa. ${ }^{7}$

- En un tercer nivel tendríamos una lectura mediada, que puede ser reseña, estudio o crítica, en tanto el objeto de la misma es presentar el texto ante un público susceptible de acudir a él en función de la valoración que del mismo se haga, o como parte de un ejercicio crítico que se presenta en medios especializados. Tenemos entonces a un lector mediador o crítico.

- En un cuarto nivel tendríamos al lector traductor, que realiza una lectura transductora, y que de forma explícita o implícita realiza las tres lecturas anteriores, para convertir un texto fuente en un texto meta. ${ }^{8}$

7 Un texto como Deirdre of the Sorrows, de John Millington Synge, puede suscitar el interés de los estudiosos de la recreación literaria de la mitología heróica gaélica, pero también el de un lingüista interesado en la modalidad diatópica del inglés que conocemos como angloirlandés, aunque un filósofo seguramente orientaría su lectura a la presencia posible del sentimiendo de la «saudade» en el mismo.

8 Doležel señala que «el prototipo de la transducción literaria es la traducción» (I999: 286), y destaca la transcendencia de las propuestas de Jirí Levy, abriendo un camino

- En el quinto nivel, el de la lectura liminar, situamos un lector liminar, que también puede aparecer implícito en la propia textualidad, pues es a él a quien se dirige el texto espectacular y ese dramaturgista ficticio del que hablamos. Tomamos esta denominación, lector liminar, de Mauro Ferraresi (Eco et al., 1995: 74-75), para hablar de un lector que se sitúa en el umbral de un nuevo proceso de semiósis, de producción significante, en el que participan los más diversos códigos, y ahí aparece la teatralidad. ${ }^{9}$ Aquí recuperamos aquella distinción de Eco (198I) entre interpretar un texto y usar un texto. Entendemos que la interpretación del texto sería la finalidad última de los lectores 2 y 3 , al ser el texto el objeto central en un trabajo hermenéutico que para el lector 4 tendría un valor instrumental, en tanto a este último le permite generar otro texto. También el lector 5 , como el 4 , hace uso del texto, aunque lo tome como pre-texto o pretexto para un nuevo proceso creativo. Ese valor de uso exige, en buena lógica, estrategias hermenéuticas orientadas a explicar y comprender el texto a través de un proceso de exégesis e interpretación.

En ese quinto nivel el texto dramático pasa a ser un producto literario susceptible de ser inte-

apenas explorado y que puede resultar especialmente relevante en la comprensión de la complejidad del acto de traducir, en tanto se entienda como mucho más que un simple trasvase lingüístico.

9 Con relativa frecuencia se tiende a confundir la dramaticidad de un espectáculo (vinculada a la conducta de los habitantes del mundo dramático y a las acciones, situaciones y conflictos que generan), con su teatralidad, pues ésta última en realidad refiere los elementos de significación utilizados en la escena y la relación que establecen entre sí. La teatralidad, que es cualidad escénica y no literaria, no proviene del texto sino que deriva del uso de los signos de todo tipo con los que se configura el mundo dramático en la escena, por lo que cabe hablar de teatralidades, en plural. 
grado en un proceso de expresión y comunicación teatral. Su lectura, análisis e interpretación se suele realizar desde el marco teórico de la Teoría general del teatro y de las disciplinas que se agrupan en su ámbito disciplinar: Escenificación, Teoría de la puesta en escena, Dramaturgia, Teoría de la interpretación, Escenografía o Tecnología teatral (Vieites, 20Io). Se trata de una lectura especializada que solo podremos realizar, con eficacia, cuando poseemos los conocimientos propios de las disciplinas señaladas, pues no se trata de un análisis literario (o no solo) sino de un análisis dramatúrgico. Las técnicas, estrategias y procesos que se ponen en marcha en este proceso de trabajo fueron analizadas, entre otros, por Hormigón (I99I), Sanchis Sinisterra (1995) o Lavandier (2003). Así, Hormigón ofrecía una tentativa metodológica que puede informar del rigor, la coherencia y la significatividad que el análisis y las estrategias dramatúrgicas pueden llegar a mostrar en relación con su objetivo último, que no es otro que orientar el proyecto y los procesos de la escenificación, es decir, de la creación teatral (Hormigón, 1991: 67):

El proceso que conduce del texto literario al espectáculo teatral es por tanto ámbito específico del trabajo dramatúrgico, ante todo en lo que respecta a la elección y compromiso con uno o varios de los sentidos profundos del texto, su revisión consecuente y el trazo estilístico en que se articularán los elementos expresivos, incluido el análisis de la funcionalidad, carácter, naturaleza, contradicciones y confrontaciones del personaje.

Ante esa articulación, Santoyo (1989) hablaba de los diferentes modelos de adaptación textual (transliteración, refundición, traslado, readaptación, reescritura, recreación, plagio, recomposición y transposición), estableciendo un abanico casi completo de las diferentes posibilidades de que dispone un director de escena o un dramaturgista (o un equipo de dramaturgia) para construir el nivel literario de un espectáculo teatral, o el propio espectáculo en su dimensión 119 virtual, cuestión también abordada por Braga Riera (20II). Y aquí aparecen numerosas preguntas y no pocos problemas ya formulados por Mateo (1995). Como señalaba Heras (I998: 25) en su justificación del espectáculo Memoria de Antigona:

Cada vez que nos enfrentamos a un texto clásico desde la dirección de escena comienzan a surgir múltiples preguntas que no tienen siempre una respuesta clara: ¿Debemos tomar el texto canónico y ser absolutamente fieles a su literatura? O por el contrario, ¿Debemos asumir ese texto y transgredir su literatura haciendo una adaptación maquillada del original? ¿Cómo ser modernos? ¿Respetando el texto pero cambiando el tiempo y el lugar? $¿ \mathrm{O}$ asumimos la arqueología y nos limitamos a una fidelidad servil de las esencias de la tradición? ¿Es posible superar hoy los magníficos montajes de esos textos que hicieron realidad los grandes maestros de la puesta en escena del siglo Xx? ¿Nos dejamos llevar por la soberbia ciega de creer que podemos hacer otra cosa o por la humildad pacata de quien piensa que tocar un clásico es casi un sacrilegio? ¿Son los clásicos nuestros contemporáneos o, realmente, necesitan una lectura actual para llegar a nuestros espectadores?

Y es ahí donde se hace tan necesario el trabajo del dramaturgista, al menos en aquella visión que de sus diversas funciones se ofrecen en Cardullo et al. (1995), Lavandier (2003), Luckhurst (2008), o en La profesión del dramaturgista (Hormigón et al., 20II). Aunque es una figura no siempre visible en muchos sistemas teatrales, su función existe, aunque sea desempeñada por otras personas, y consiste en crear un nuevo texto que denominamos texto dramatúrgico, que es aquel del que parte todo el equipo artístico 
para comenzar sus trabajos de realización teatral, y que contiene el texto traducido, sometido a revisión y adaptación de ser el caso.

120
En efecto, en todo proceso de creación teatral se formula, de forma explícita o implícita, un espectáculo ideal que se configura en un texto dramatúrgico, que dará lugar a un texto de la puesta en escena y que se concreta en el texto teatral del espectáculo histórico, aquel que el director determinará y fijará con su equipo durante el proceso de escenificación y en los últimos ensayos generales; y ese espectáculo histórico será actualizado, con pequeñas y a veces insignificantes variaciones, en las diferentes funciones. Ese es el espectáculo real y concreto que el espectador contemplará en un día particular y que es una variación del espectáculo bistórico que el director, el equipo de realización y producción y el elenco decidieron dar por versión definitiva. $Y$ cuando hablamos de texto no referimos únicamente la dimensión puramente lingüística del espectáculo, sino la suma de elementos de significación (Kowzan, 1969) utilizados en la configuración sistémica específica de ese espectáculo concreto, y que determinan su teatralidad (Martínez, 1989).

El dramaturgista, dramaturg en la propuesta de Lessing en su Hamburgische Dramaturgie, ${ }^{\text {,o }}$ partiendo del trabajo del traductor (a veces con su ayuda, y otras veces siéndolo él mismo), altera estructuras, varía el número de escenas, actos, cuadros o personajes, mezcla diálogos y somete

Io Existe versión española, publicada en su segunda edición de 2004 por la Asociación de Directores de Escena de España, con traducción de Feliú Formosa: Dramaturgia de Hamburgo. Lessing distingue entre el «dramatiker», autor de obras dramáticas, y el «dramaturg», que adapta, asesora, elabora repertorios y colabora activamente en el trabajo vinculado con la dimensión literaria de la creación escénica. No cabe confundir al dramaturgo, autor de obras dramáticas («playwright» en inglés), con ese «dramaturg», y por ello en castellano se habla de «dramaturgista». el texto (o textos) a ese proceso necesario de reescritura y adaptación al contexto del grupo de trabajo y de los receptores virtuales, como señalaba Pavis en su definición de dramaturgia, entendiendo que «designa el conjunto de opciones estéticas e ideológicas que el equipo de realización, desde el director hasta el actor, ha tenido que realizar», por cuanto siempre tiende «a superar el marco de un estudio del texto dramático, para abarcar texto y realización escénica» (1990: 157). Así llegamos a las razones que a veces se esgrimen para trabajar con versiones y adaptaciones que, en ocasiones, suelen diferir del texto traducido. Pues si en los textos dramáticos escritos en la misma lengua en que se realiza el espectáculo la dramaturgia siempre es necesaria, en tanto se hace necesario adaptar un material lingüístico a un contexto escénico y sociocultural, más necesaria todavía resulta cuando se trata de textos traducidos (Mateo, I995: I05):

Due to the close communication between addresser and addressee in the theatre, plays are usually subject to alterations so as to fit the established theatrical conventions and cultural expectations of the target audience. This and the fact that spoken language changes more quickly than written explain why there are more often translations of the same play, as every new generation would ideally require a new translation with which to share a new experience.

Como señalaba González Doreste (I995: 415) a propósito de la traducción de un texto del dramaturgo franco-argentino Copi:

(...) no podíamos limitarnos a realizar una traducción fiel, sino que debía ser, además, una actualización, una adaptación, que respondiera a las exigencias socioculturales del público actual, sin alterar para ello en ningún momento la estructura dramática del texto. 
Pavis situaba, en la misma dirección, las primeras dificultades de la escenificación de un texto escrito en otra lengua en su traducción, pues «we cannot simply translate a linguistic text into another; rather we confront and communicate heterogeneous cultures and situations of enunciation that are separated in space and time» (1992: I36). Son problemas derivados de la necesaria adecuación pragmática entre texto y contexto (Scolnicov, Holland et al., 1989; Mateo, 2006), o entre texto, espectáculo y audiencia (Zuber-Skerritt et al., I984; Törnqvist, I99r; Jaccomard, 20I3). Problemas ante los que el traductor debiera ser un necesario soporte en los procesos de dramaturgia y escenificación, pues su participación en el proceso de diseño y creación espectacular permite «trabajar en colaboración con el director y los actores», y genera una dinámica «muy enriquecedora» (González Doreste, I995: 423; Puga, 20I2), pero contribuye además a la excelencia del producto final, cuestión sobre la que vale la pena insistir. En esa dirección, traducción y dramaturgia se complementan, como señala Johnston al reclamar al traductor una mayor «dramaturgical awareness» (2013: 382).

\section{DE LA TRADUCCIÓN A LA ESCENIFICACIÓN: TEXTOS Y PROCESOS}

En la relación entre teatro y traducción es habitual diferenciar dos ámbitos fundamentales: aquel que se vincula con la traducción puramente literaria, y aquel que se vincula con la traducción escénica, que no por ello deja de ser literaria. En la primera, que el profesor Santoyo definiera como reader-oriented (1989: 97), el trasvase se realiza de página a página, en tanto en la segunda, que Santoyo define como performance- oriented (I989: 97), ${ }^{\mathrm{II}}$ el trasvase se realiza entre página y escena, pero igualmente entre la escena, mundo de la emisión, y la sala, mundo de la recepción, sin olvidar el caso de los espectáculos 121 que cuentan con una traducción subtitulada, mediación presente en determinados teatros $\mathrm{y}$ festivales. Tampoco podemos olvidar el ensayo teatral, particularmente aquella literatura científica o divulgativa que es propia de las disciplinas sobre las que se conforma la praxis teatral, desde la interpretación a la dirección escénica, o aquella que acompaña la apropiación de una autora y que puede funcionar como «paratexto» en tanto su misión sea (re)contextualizar autora y obra. ${ }^{\mathrm{I2}}$

En un nivel teórico, derivado del análisis empírico de la práctica propia y ajena (Vieites, 2or6b, 20I6c) y de la conocida propuesta de Pavis (1992), pero también considerando lo que aporta Nord (2005), podemos decir que en todo proceso de creación teatral, incluso en aquellos espectáculos en los que el componente textual y verbal es escaso o nulo, debiéramos considerar, cuando menos, los siguientes «textos»:

- Texto Dramático / Texto [fuente] (TI). Se trata de la obra original, aunque cabe señalar que no siempre estamos ante un texto dra-

II El hecho de que esa diferencia exista, como se constata en un análisis empírico del corpus de textos traducidos (publicados o no), no invalida el hecho de que, siquiera en el plano teórico, estemos ante una diferencia que no se sostiene, al menos en el caso del texto dramático, si consideramos que un tal texto está integrado en gran medida por actos de habla, aunque en determinadas épocas lleguen cargados de retórica. A la persona responsable de la traducción compete reproducir un proceso de comunicación, porque traducir es más que un tránsito de palabras y oraciones entre lenguas, ya que implica operar con discursos que se entrelazan, especialmente en su dimensión pragmática $(\mathrm{Ju}-$ det de la Combe, 2013).

I2 Un buen ejemplo es el Ciclo Autor, coordinado por Vicente León en el Teatro Pradillo de Madrid y que permitió presentar autoras como Sarah Kane o Caryl Churchil, o autores como Michel Azama o David Hare, en una triple perspectiva: la escénica, la académica y la crítica (Díaz Díaz, 2004). 
mático sino ante otro material, de carácter literario o no. En ocasiones se parte de poemas, cuentos, narraciones varias, novelas, documentos históricos, material de prensa y similares.

- Texto Dramático / Texto traducido [meta] $\left(\mathrm{T}_{2}\right)$, que en ocasiones también puede ser Texto Dramático / Texto publicado, pues en España contamos con una sólida tradición de traducción literaria de obra dramática para el mercado editorial, con colecciones tan populares como las de Escelicer, Austral o Cátedra Letras Universales, entre otras muchas. Aquí finalizaría el recorrido de la traducción exclusivamente literaria. El texto traducido que se toma como pretexto de la creación escénica ya puede existir como tal, esté publicado o no, o puede derivar de una «encarga» de traducción.

- Texto Dramatúrgico $\left(\mathrm{T}_{3}\right)$, o propuesta textual para el proyecto general del espectáculo, que surge de un $\mathrm{T}_{2}$ ya existente o expresamente encargado. En sistemas teatrales desarrollados este $T_{3}$ es elaborado por el departamento de dramaturgia del teatro o por el dramaturgista de la compañía, cuando existe. En ocasiones el $\mathrm{T}_{3}$ no toma como texto previo un $\mathrm{T}_{2}$ integro y original, sino un $\mathrm{T}_{2}$ ' que puede ser generado por el equipo de creación como versión o adaptación de uno o varios $\mathrm{T}_{2 .}{ }^{\mathrm{I}}$ Aunque este $\mathrm{T}_{2}$ ' en otras ocasiones parte de un $\mathrm{T}_{2}$ en otra lengua con lo que entramos en el ámbito de las traduc-

I3 Suele ocurrir esto con espectáculos que parten de textos de autores o autoras de los que hay traducción disponible, una o varias, y en los que el texto dramatúrgico se elabora sin tener en cuenta a las personas que detentan la autoría de esas versiones, incluso mezclando versiones, con lo que se consigue una mayor opacidad del texto final y permite que el responsable de la versión pueda registrar la traducción a su nombre, sea de Shakespeare, sea de Brecht, a veces sin conocimiento de la lengua en que escriben. ciones indirectas, práctica bastante habitual en dominios lingüísticos próximos. ${ }^{14}$

- Texto de la Puesta en Escena $\left(T_{4}\right),{ }^{15}$ que se elabora y se fija a partir del anterior $\mathrm{T}_{3}$ en los procesos de construcción de cada situación, del personaje, de la acción; o en los ensayos de la escenificación, que siempre conllevan cambios y adecuaciones ${ }^{16}$ derivadas de la emisión, la elocución, la prosodia, la sonoridad, los ritmos, los tiempos y los tempos, o incluso el espacio sonoro considerado en su conjunto (Espasa, 2009).

- Texto Teatral $\left(T_{5}\right)$, que se fija tras los ensayos generales y se emite, normalmente con mínimas alteraciones, o ninguna, en la actualización del espectáculo en cada función concreta, y es el texto que finalmente llega al espectador. El estudio de los tránsitos que

I4 Así un director o dramaturgista que conozca el francés puede decidir generar un $\mathrm{T}_{3}$ en castellano de la obra de Pinter Ashes to Ashes a partir de la versión francesa de Éric Kahane, o de una versión portuguesa o italiana. Infelizmente se trata de una práctica más habitual de lo normal (VV. AA., 20I4).

I5 En mi opinión es muy importante diferenciar entre este proceso de reescritura y la traducción propiamente dicha, pues si bien es cierto que en no pocas ocasiones el equipo de creación «reescribe» el texto, y a veces de forma notable (y en consecuencia lo adaptan, lo versionan, lo deconstruyen, lo manipulan...), esa dramaturgia en proceso parte de un texto ya traducido, no del texto fuente, con lo que estamos ante una «dramaturgia de la dramaturgia» (del $\mathrm{T}_{3} \mathrm{al}_{4} \mathrm{~T}_{4}$ a partir de un $\mathrm{T}_{3}$ ' «in progress»). Es cierto, como señala Aaltonen que «all theatre practitioners are involved, to some degree, in the rewriting of a text for a particular production» (2013: 385 ), pero esa reescritura parte del texto traducido, y obedece a un proceso nuevo en el que operan otras disciplinas artísticas que ejercen actrices, actores, escenógrafas, directoras, iluminadores o dramaturgistas, pues ellas y ellos son los dichos «practitioners».

I6 Se trata de un texto en proceso que sufre variaciones debido a factores muy diversos, incluso a cuestiones puramente técnicas, como las dimensiones del escenario que afectan al tránsito espacial de los personajes, o a la necesidad de eliminar un personaje, o fundir varios en uno solo, debido al número de personas que conforman el elenco. 
se producen entre $\mathrm{T}_{\mathrm{y}}$ y $\mathrm{T}_{5}$ es un ámbito muy interesante de investigación, también en el ámbito de la historia de la traducción, en tanto informan de procesos y estrategias de adecuación y apropiación de textos en las perspectivas cultural, artística, lingüística, semántica o pragmática, pero también en la económica o la profesional (con todas las malas prácticas y las cuestiones éticas señaladas). ${ }^{17}$

Tradicionalmente el traductor es el responsable del T2, y ahí suele terminar su tarea, si bien hay directores o directoras que promueven su participación activa en la elaboración del $\mathrm{T}_{3}$ y en el seguimiento y adecuación del $\mathrm{T}_{4}$, e incluso en la fijación del $\mathrm{T}_{5} \cdot{ }^{\mathrm{I}}{ }^{8}$ Cuando el traductor participa en ese proceso de construcción y creación del espectáculo, su rol puede variar considerablemente, desde hacer una traducción según el canon tradicional para luego participar en la explicación e interpretación de la forma y del sentido, o participar activamente desde el inicio en el proceso de reescritura, manipulación o transgresión textual. Aaltonen (I995: 9I-92) afirmaba en esa dirección:

(...) not all translators are granted equal power by the theatrical system... The first category of translators are those whose only connection with the stage is the translation work... Their role is seen as that of mediators rather than of creators... The second category

17 En ocasiones es este el texto que se publica, lo que puede tener interés para unha historia de la dramaturgia, si bien deja de ternerlo cuando el sistema literario de acogida carece de una versión integra del texto fuente. Infelizmente hay muchos ejemplos.

I8 Un ejemplo del proceso completo, considerado como una buena práctica, es el caso de Oleanna, espectáculo creado por el director Manuel de Benito a partir del texto homónimo de David Mamet con traducción de Juan V. Martínez Luciano, producido por el Teatro Español de Madrid en 20II (Mamet, 20II). are translators who work within the theatre, such as dramaturges or directors... They can, if they so wish, make adjustments or interpret the text according to need.

Es importante no perder de vista esta argumentación, dado que el traductor literario y el traductor literario-escénico trabajan con perspectivas y finalidades diferentes, ya que el producto de su trabajo difiere de forma considerable, aunque el resultado inicial en tanto traducción de actos de habla debiera ser siempre el mismo. En el primer caso, el texto es esencial en cuanto objeto fuente y meta, pero en el segundo el texto no deja de ser un pretexto en la creación del espectáculo, finalidad última del proceso. Eso explica, si bien no justifica, ${ }^{19}$ el hecho de que en algunos países se prescinda del traductor en la realización de la versión inicial o final del texto en la lengua meta, o que sean tan comunes las traducciones indirectas, en tanto se buscan conflictos, situaciones, motivos, tópicos, conductas, acciones, pautas psicofísicas, formas de habla, de entre las múltiples opciones que la cultura meta nos ofrece en cada caso. Son, estas últimas, versiones que no buscan tanto la literalidad o la literariedad cuanto un palimpsesto construido a base de argumento y diálogos. La proximidad entre lenguas como el castellano, el italiano, el francés o el portugués facilita esta forma de hacer. ${ }^{20}$

Considerando el tránsito que va de $\mathrm{T}_{\mathrm{I}}$ (texto fuente) a $\mathrm{T}_{5}$ (aquel texto que oímos y vemos en una función en un teatro concreto), las casuísti-

I9 El hecho de explicar y considerar el texto traducido como «pre-texto» o «pretexto», en ocasiones no deja de ser un pobre pretexto, o excusa, para operar con una libertad plena en una manipulación textual que a veces implica una total impunidad.

20 Como antes se decía, en estos casos se prima, siguiendo a Hjelmslev, la substancia del contenido frente a la forma y la substancia de la expresión y la propia forma del contenido. 
cas pueden ser múltiples, también si consideramos a los agentes que participan en el proceso y sus funciones, pues ni todos los teatros ni todas 124 las compañías cuentan con un dramaturgista en plantilla ni con su colaboración temporal, y a veces ni siquiera con un director de escena, ausencias y carencias que no invalidan el modelo propuesto. Pero de entre todos los casos posibles son tres los que reclaman nuestra atención, al no constituir ejemplos de buenas prácticas:

I. En esta primera opción el texto ya está traducido, y disponible en tanto publicado por vías diversas (ahora cada vez más en portales específicos), ${ }^{21}$ antes de que una compañía decida crear con él un espectáculo. ${ }^{22}$ Cabe entonces contar, o no contar, con la colaboración de la persona responsable de la traducción para la realización de la dramaturgia, y entonces las compañías gestionan la cesión de derechos del autor y del traductor (o no, en cuyo caso estaríamos ante una conculcación de derechos muy común), ${ }^{23}$ e inician su trabajo ajenas a cualquier consideración que este último pudiera hacer. Craso error,

${ }^{21}$ Véase como muestra la colección Bivir, o biblioteca virtual de traducción de la Asociación de Tradutores Galegos: http://tradutoresgalegos.com/bivir.html

${ }_{22}$ Con los clásicos la picaresca ofrece una casuística considerable, aunque la forma más habitual de apropiación de derechos consiste en que alguien, sin conocer siquiera la lengua original, realice una versión a partir de las traducciones disponibles, que se registra como propia en las instancias que correspondan. Una práctica muy recurrente con la obra de Shakespeare, que incluso lleva al cartel del espectáculo la autoría de la versión señalando el nombre de una persona incapaz de articular dos frases en inglés.

${ }_{23}$ Infelizmente suele ser una práctica bastante común partir de una traducción publicada, sobre la que algún integrante de la compañía, sea el director o no, realiza su versión, introduciendo aquellas modificaciones necesarias para disimular el plagio, práctica que sitúa a los traductores no solo ante una flagrante usurpación de derechos, sino en una situación de absoluta indefensión. Es evidente que esa práctica obedece en muchos casos al deseo de cobrar los derechos de traducción y/o versión. pues si hay una persona que conoce a fondo el texto es aquella que lo ha traducido, dado que la traducción exige un proceso de exégesis y hermenéutica textual (Nord, 2005) que en muy escasas ocasiones las compañías de teatro están en condiciones de abordar, especialmente en todo lo que atañe a la forma y la substancia de la expresión, a la dimensión más lingüística y estilística del texto, o en cuestiones mucho menos sutiles que informan del despropósito a que se puede llegar. ${ }^{24}$ De nuevo es importante insistir en que en la traducción de un texto dramático no solo se «transduce» un mundo, sino procesos comunicativos.

2. En la segunda opción, el texto no está traducido y se busca una persona para realizar la traducción, que puede ser persona con capacidad y competencia, o no, y en bastantes ocasiones no lo es, con lo que la traducción, aún siendo directa, ${ }^{25}$ puede acabar por ser una pobre versión del texto original, en la que los personajes usan el mismo registro y no se caracterizan por su forma de hablar, ni en su expresión muestran signos de su carácter, error sumamente habitual. ${ }^{26} \mathrm{Si}$ esta última posibilidad es

${ }^{24}$ La obra de Eugene O'Neill, Long Days's Journey into Night, se suele traducir como Largo viaje hacia la noche; $\mathrm{y}$ ¿de quién?, cabría preguntar, pues en realidad en su título el autor expresa el tránsito terrible que viven los personajes entre la luz y la paz (el día) y la oscuridad y el tormento (la noche). Se pueden aducir razones comerciales para el despropósito, pero lo cierto es que O’Neill no tituló su obra Long Journey into Night.

${ }_{25}$ En comunidades en las que existen dos lenguas oficiales, es habitual la práctica de la traducción indirecta, con lo que la versión que llega se realiza desde la traducción en castellano o portugués. En el caso de los clásicos, Shakespeare, Molière, Brecht, Ionesco, es una práctica mucho más común de lo aconsejable.

${ }_{26}$ Un buen ejemplo lo tendríamos en las obras de Martin McDonagh, en especial las ambientadas en la Irlanda atlántica, en las que los personajes se caracterizan en sus idiolectos, en su praxis lingüística, que no siempre se reconocen en la traducción. Un caso excepcional es el de 
reprobable desde cualquier punto de vista, en la primera el trabajo encargado al traductor competente suele finalizar con la entrega de su versión, renunciando la compañía a todo cuanto el traductor puede aportar, al menos en la conversión del $\mathrm{T}_{2}$ (texto traducido) en $\mathrm{T}_{4}$ (texto de la puesta en escena que supone una varianza permanente del texto dramatúrgico o $\mathrm{T}_{3}$ ). Por ello es tan aconsejable la participación del traductor o traductora al menos en el tránsito del $\mathrm{T}_{2}$ al $\mathrm{T}_{4}$.

3. En la tercera de las opciones, el texto de trabajo (la generación de un $\mathrm{T}_{2}$ para ser $\mathrm{T}_{3}$ ) no se trae a la lengua meta desde su fuente $\left(\mathrm{T}_{\mathrm{I}}\right)$ $y$ en consecuencia desde un $T_{2}$ original, sino desde otra traducción $\left(\mathrm{T}_{2}{ }^{\prime}\right)$, realizada por un traductor o por persona que conoce ese otro idioma al que está traducido el texto fuente, $y$ en ese proceso el texto se somete a una pérdida constante de matices en la forma y la substancia de la expresión y del contenido. Aquí aparecen las traducciones indirectas, más comunes de lo deseable. ${ }^{27}$

Un análisis sistemático y comparado de estas tres posibilidades mostrará casos de buenas prácticas, los menos, y casos de prácticas desaconsejables, los más, y su documentación y estudio resulta cada vez más necesario. Pero también mostrará que en muchos casos el trabajo del traductor es realmente invisible,

la traducción de The Tinker's Wedding de John Millington Synge, que Xepe Casanova tradujo al gallego como $A$ boda do latoeiro para la Compañía Marías, en la que el traductor reproduce el habla de los moinantes gallegos de la zona de Carballo (La Coruña) como elemento caracterizador y con excelentes resultados.

27 En un trabajo anterior (Vieites, 2015) analizábamos el impacto de tales prácticas en Galicia, pero también el tránsito progresivo a una mejor praxis profesional que se produce en los últimos años, a la que ha contribuído la existencia de estudios de traducción en la Universidad de Vigo. cuando en realidad su aportación podría ser especialmente sugerente y estimulante para el equipo de creación, sobre todo pensando que su integración en ese equipo sería a su vez un estímulo para su propio desempeño profesional y un apoyo necesario en la construcción de la dramaturgia del espectáculo.

\section{CONCLUSIONES}

$\mathrm{El}$ «giro escénico», que proponemos como un marco para estudiar las relaciones entre teatro y traducción, nos permite identificar de una forma precisa los agentes, los procesos y las prácticas que cabe considerar en ese tránsito que va de la página a la escena. $Y$ aparecen entonces dos figuras importantes en otros sistemas teatrales que en el nuestro gozan de poca presencia y de escasa visibilidad: la responsable de la traducción y la responsable de la dramaturgia, ambas esenciales en los procesos de escenificación.

Dadas las características del sistema teatral español, y de sus prácticas artísticas, la traducción de textos dramáticos para la creación teatral o la utilización de textos dramáticos ya disponibles en el mercado editorial, presenta una serie de disfunciones que también informan del escaso nivel de legitimidad del oficio del traductor de obra dramática en el campo teatral, y de cómo los profesionales de este último parecen justificar determinadas malas prácticas (como la de utilizar varias traducciones para elaborar un texto definitivo de trabajo sin consultar el texto original) al centrar su atención en lo que Hjelmslev definía como substancia del contenido: el mensaje. Por eso tal vez fuese necesario comenzar a implementar programas de gestión de la calidad en los procesos de creación escénica para asegurar que el diseño general y la toma de decisiones se ajusta a un patrón asentado en una guía buenas prácticas que también habría 
que construir (Vieites, 2007), y en las que se debiera considerar la presencia de una traductora y de una dramaturgista en el desarrollo del proyecto. $^{28}$

Este giro escénico tiene además, a nuestro entender, importantes aplicaciones tanto en la docencia como en la investigación. En el primer caso porque nos permite ejemplificar de forma precisa lo que es el texto dramático, sus voces, sus posibilidades de lectura, y los diferentes textos a que da lugar en los procesos de escenificación, pero considerando igualmente los agentes implicados, sus intereses, sus funciones y responsabilidades, y proponer al alumnado interesantes estudios de campo en búsqueda de ejemplos que expliquen la casuística. En el segundo caso porque permite analizar ámbitos hasta ahora muy poco explorados en los estudios propios del campo, como hemos mostrado en algún trabajo reciente (Vieites, 20r6b, 20r6d), en el que se vinculan traducción y agentes del campo de la creación escénica como compañías de teatro o directoras de escena, lo que permite ofrecer una visión más compleja de las relaciones entre traducción y teatro.

Por volver al principio, diremos que el «Código de buenas prácticas para traducciones y adaptaciones teatrales», suscrito entre ACETraductores y la Asociación de Directores de Escena de España, plantea cuestiones sencillas pero substantivas en relación con derechos $\mathrm{y}$ deberes de personas que ejercen profesiones que no han logrado un grado de visibilidad,

${ }^{28}$ Aún contando con un texto traducido y solicitando todos los permisos, siempre es necesario conocer el texto fuente y sus características, y por ende trabajar con una traductora. Pues podría darse el caso de una traducción del texto de Harold Pinter antes señalado, The Room, en el que se hubiese obviado, o minimizado, toda la carga ilocutoria y perlocutoria que el texto contiene y el fragmento muestra, con lo que la dimensión relacional y pragmática se diluiría, y el espectáculo sería un acto fallido. legitimidad o institucionalización suficiente para garantizar un ejercicio pleno de las mismas. Por ello se establecen «algunas normas que sirvan de guía a directores, productores, autores teatrales, traductores y cuantos participan en la puesta en escena de obras literario-dramáticas, a fin de mantener el necesario nivel ético de sus respectivas profesiones» (ACE/ADE, 20I4: 33). Normas que invocan un sentido común tantas veces conculcado, y que en buena medida se formulaban en las preguntas que acompañaban la mesa de trabajo que se reproducía en las páginas de la revista: «¿Se reconoce el trabajo de los traductores en los trabajos escénicos de nuestro país? ¿Con qué libertad o permiso se hace uso de las traducciones en los espectáculos españoles?» (VV. AA., 2014: I5).

Pero, además de las cuestiones éticas y deontológicas, no podemos olvidar las profesionales, y la sociología de las profesiones (Sánchez Martínez, Sáez Carreras y Svensson, 2003) nos enseña que el ejercicio de una profesión supone, entre otras cosas, poseer un conocimiento especializado de un campo concreto y de las actividades que le son propias y diferenciales. El hecho de que en determinados ámbitos profesionales el acceso al ejercicio no esté regulado garantiza que personas dotadas de capacidad, habilidad y talento no precisen de otras armas que su trabajo para mostrar su valía, pero este tipo de personas no abundan. Se hacen necesarios entonces los centros de formación, para desarrollar capacidades y habilidades, para generar y provocar talento, pero también para tomar conciencia de que a pesar de que existan notables artistas multidisciplinares (pocos en realidad), la división del trabajo permite el desarrollo de procesos de especialización y mejora que redundan en calidad y excelencia. Por eso es importante señalar que del mismo modo en que un actor o un director de escena poseen un 
conocimiento muy especializado que se vincula a competencias muy específicas en el saber, el hacer, en el hacer el saber o en el saber el hacer, también el traductor posee idénticas competencias aplicadas a su propio campo. Y del mismo modo en que un traductor no asume las funciones del actor o del director de escena, estos, por muchos idiomas que sepan, no debieran asumir las funciones de aquel, porque traducir implica mucho más que saber un idioma o saber escribir con soltura (si es que a tanto se llega).

El traductor debiera ser, con el dramaturgista, una figura importante en el proceso de diseño y realización de un espectáculo asentado en un texto tomado de otra lengua, no solo porque el citado «Código» recomiende que «las alteraciones en una traducción deberán contar siempre con el consentimiento del traductor», o porque se entienda que el traductor pueda o deba «participar en la adaptación o seguimiento de los ensayos, acordando la remuneración adecuada» (ACE/ADE, 2OI4: 33), sino porque entendiendo el espectáculo teatral como acto complejo de comunicación con emisores múltiples, todas las voces y todas las formas del saber y del hacer (indudablemente también del ser, en su dimensión ética) son necesarias y ayudan a enfrentar la complejidad, sin olvidar que «una representación es un producto final altamente organizado de un proceso y de una organización complejos» (Sarason, 2002: 35).

Finalmente, en la intersección entre traducción y teatro que provoca la traducción para la escena, en tanto espacio de la escenificación, en ese tránsito page to stage (Zuber-Skerritt et al., I984), se hace necesario considerar territorios de confluencia que debieran comenzar en los procesos de formación, especialmente en la transversalidad de unos contenidos capaces de generar competencias vinculadas con la comprensión de los fenómenos culturales como hechos complejos en el que todos los agentes son necesarios y ninguno prescindible, potenciando así una visión molar, global o sistémica de la realidad social, en la que se inscriben los 127 campos cultural y artístico (Martínez, I989).

Para promover esa visibilidad del traductor como agente necesario en la creación escénica asentada en la obra traducida, se precisa generar en el mundo de la traducción y en el mundo de la escena, una visión positiva de tal rol, de esa función. Y una tal visión debiera comenzar a conformarse en las Facultades de Traducción e Interpretación y en las Escuelas Superiores de Arte Dramático o Facultades de Artes Escénicas, tanto en la transversalidad de las diferentes materias que integran el currículum en cada caso, como en el nivel de las competencias terminales que se debieran vincular a contenidos específicos, pero también celebrando encuentros periódicos para analizar tantas cuestiones comunes como hemos señalado, y para los que no se requieren más medios que la voluntad de organizarlos. Cabría también pensar en introducir en el plan de estudios de sus titulaciones alguna disciplina orientada tanto a ofrecer al traductor una formación básica en dramaturgia, como asentar en el creador escénico una lectura del tránsito que va del texto a la escena, y de las operaciones que ese tránsito implica, de los agentes más capacitados en cada caso, e igualmente una comprensión real y positiva del trabajo interdisciplinario, que siempre conduce a la excelencia. Y finalmente establecer un protocolo de buenas prácticas para que todos los agentes invocados en este trabajo puedan desarrollar plenamente su actividad. Por el bien común de creadores, mediadores y receptores.

RECIBIDO EN MARZO DE 2015 ACEPTADO EN ABRIL DE $20 I 6$ VERSIÓN FINAL DE MARZO DE 2017 


\section{BIBLIOGRAFÍA CITADA}

Aaltonen, Sirkku (1995): «Translating plays or baking apple pies: A functional approach to the study of drama translation». En: M. Snell-Hornby, Z. Jettmarová y K. Kaindl (eds.) Translation as Intercultural Communication. Amsterdam: John Benjamins, pp. 89-98.

- (2000): Time-sharing on stage. Drama Translation in Theatre and Society. Clevedon: Multilingual Matters LTD.

- (2013): «Theatre translation as performance». Target, 25 (3), pp. 385-406.

ACE/ADE (20I4): «Código de buenas prácticas para traducciones y adaptaciones teatrales». $A D E /$ Teatro, 153, p. 33 .

Arqués, Rossend (2015): «Les traduccions teatrals de Vallespinosa». Quaderns. Revista de Traducció, 22, pp. 75-94.

Austin, John Langshaw (I982): Cómo hacer cosas con palabras. Barcelona: Paidós.

Barthes, Roland (I967): Ensayos criticos. Barcelona: Seix Barral.

Bassnett, Susan (I99I): «Translating for the Theatre. The Case Against Performability». Languages and Cultures in Translation Theories, 4 (I), pp. 99-III.

- (2002): Translation Studies (3rd Edition): London: Routledge.

- (20I2): «Translation studies at a cross-roads». Target, 24 (I), pp. 15-25.

Bassnett, Susan y Lefevere, André (eds.) (I998): Constructing Cultures: Essays on Literary Translation. Clevedon: Multilingual Matters LTD.

Beckett, Samuel (1990): The Complete Dramatic Works. London: Faber and Faber.

Bobes Naves, María del Carmen (1987): Semiología de la obra dramática. Madrid: Taurus.

Braga Riera, Jorge (20II): «¿Traducción, adaptación o versión?: maremágnum terminológico en el ámbito de la traducción dramática». Estudios de Traducción, I, pp. 59-72.

Burke, Kenneth (I945): A Grammar of Motives. New York: Prentice Hall.

Buzelin, Hélène (2005): «Unexpected Allies». The Translator, II (2), pp. I93-2I8.

Cardullo, Bert (ed.) (1995): What is dramaturgy? New York: Peter Lang.

Carlson, Marvin (2003): Performance. New York: Routledge.

Curell, Clara (2013): «La traducción literaria como práctica pedagógica». Onomázein, 28, pp. I-I3.

Díaz Díaz, Isabel M. (2004): "Los montajes del Ciclo Autor: el aire que nos devora». Primer Acto, 303, pp. 39-45.

Doležel, Lubomir (1999): Heterocósmica. Ficción y mundos posibles. Madrid: Arco Libros.

Eco, Umberto (198I): Lector in fabula. La cooperación interpretativa en el texto narrativo. Barcelona: Lumen.

- (ed.) (I995): Interpretación y sobreinterpretación. Cambridge: Cambridge University Press.

Escandell Vidal, María Victoria (2005): La comunicación. Madrid: Gredos.

Espasa, Eva (2009): «Repensar la representabilidad». Trans. Revista de Traductología, I3, pp. 95-I05.

Evreinov, Nikolai (1927): The Theatre in Life. New York: Brentano.

Ezpeleta, Pilar (2007): Teatro y traducción. Madrid: Cátedra.

- (2009): «Introducción. De la traducción teatral». Trans. Revista de Traductología, I3, pp. II-I7.

Fábregas, Xavier (1975): Introducción al lenguaje teatral. Barcelona: Los Libros de la Frontera.

Fernández Cardo, José María (I995): «De la práctica a la teoría de la traducción dramática». En: F. Lafarga y R. Dengler (eds.) Teatro y Traducción. Barcelona: Universitat Pompeu Fabra, pp. 4054I2.

García Barrientos, José Luis (I99I): Drama y tiempo. Madrid: csic.

Garrido Vilariño, Xoán Manuel (2004): «Texto e paratexto. Tradución e paratradución». Viceversa, 9/ı, pp. 3i-39.

Genette, Gérard (1987): Seuils. Paris: Éditions du Seuil.

Ghignoli, Alessandro (20I3): «La traducción teatral o la endíadis de un texto. Una reflexión comparada». Mutatis Mutandis, 6 (2), pp.321-329.

Goffman, Erving (1959): Presentation of Self in Everyday Life. New York: Anchor Books.

- (1974): Frame Analysis. New York: Harper and Row.

González Doreste, Dulce María (I995): «El teatro de Copi: traducción y recepción». En: F. Lafarga y R. Dengler (eds.) Teatro y Traducción. Barcelona: Universitat Pompeu Fabra, pp. 4I3-424.

Grotowski, Jerzy (1970): Hacia un teatro pobre. México: Siglo XXI. 
Heras, Guillermo (ı998): «Memoria de memorias». En vv. AA. Memoria de Antígona. Compostela: IGAEM, pp. 2I-29.

Hickey y Pellizzoni, Margarita (I789): Poesías varias sagradas, morales y profanas o amorosas: con dos poemas épicos, tomo I. Madrid: Imprenta Real.

Hjelmslev, Louis (1972): Ensayos Lingüísticos. Madrid: Gredos.

Hormigón, Juan Antonio (1991): Trabajo dramatúrgico y puesta en escena. Madrid: Publicaciones de la ADE.

- (ed.) (20II): La profesión del dramaturgista. Madrid: Publicaciones de la ADE.

Jaccomard, Hélène (2013): «Text Versus Stage: The Case of Yasmina Reza's Art». Australian Journal of French Studies, 50 (2), pp. 232-245.

Johnston, David (2013): «Professing translation. The acts-in-between». Target, 25 (3), pp. 365-384.

Judet de La Combe, Pierre (2013): «Teatro, sintaxis y traducción». Mutatis Mutandis, 6 (2), pp. 487-508.

Kowzan, Tadeusz (1969): «El signo en el teatro». En: VV. AA. El teatro y su crisis actual. Caracas: Monte Ávila, pp. 25-6o.

Lapeña, Alejandro L. (20I4): «Recalificar el páramo. Bases para un nuevo modelo traductológico del análisis del texto teatral». Sendebar, 25, pp. I49-I72.

Lavandier, Yves (2003): La dramaturgia. Madrid: Ediciones Internacionales Universitarias.

Luckhurst, Mary (2008): La palabra que empieza por $D$. Madrid: Fundamentos.

Mamet, David (2oII): Oleanna. Madrid: Teatro Español.

Marinetti, Cristina (2013): «Translation and theatre. From performance to performativity». Target, 25 (3), 307-320.

Martínez, Miguel (1989): Comportamiento bumano. Nuevos métodos de investigación. México: Trillas.

Martínez Paramio, Agapito (2006): Cuaderno de dirección teatral. Ciudad Real: Naque.

Mateo, Marta (1995): "Translation strategies and the reception of drama performances: a mutual influence». En: M. Snell-Hornby, Z. Jettmarová y K. Kaindl (eds.) Translation as Intercultural Communication. Amsterdam: John Benjamins, pp. 99-IIO.

- (2006): «Successful strategies in drama translation: Yasmina Reza's Art». Meta, 5I (I), pp. I75-183.

Melendres, Jaume (I999): «Los padres de la criatura». $A D E /$ Teatro, 76, pp. 36-4I.
Miras, Domingo (1985): Las alumbradas de la Encarnación Benita. Madrid: García Verdugo.

Nord, Christiane (2005): Text Analysis in Translation: Theory, Methodology, and Didactic Application of a Model for Translation-Oriented Text Analysis (2nd Edition): London: Routledge.

Pavis, Patrice (1990): Diccionario del teatro. Dramaturgia, estética, semiología. Barcelona: Paidós.

- (1992): «Toward specifying theatre translation». En: P. Pavis, Theatre at the crossroads of culture. London: Routledge, pp. I36-I59.

Pérez-Rasilla, Eduardo (2008): «Breves consideraciones en torno a la teoría y la práctica de la dramaturgia en España». En: M. Luckhurst, La palabra que empieza por D. Madrid: Fundamentos, pp. 215-239.

Pinter, Harold (1996): Plays I. London: Faber and Faber.

Puga, Ana Elena (2012): "Translation and performance: a preview of Theatre Survey's first working session». Theatre Survey, 53, pp. 309-316.

Sánchez Martínez, Mariano; Sáez Carreras, Juan y Svensson, Lennart (2003): Sociología de las profesiones. Pasado, presente y futuro. Murcia: Diego Martín.

Sanchis Sinisterra, José (I995): «Por una dramaturgia de la recepción». ADE/Teatro, 4I-42, pp. 64-70.

Santoyo, Julio César (I989): «Traducciones y adaptaciones teatrales: ensayo de tipología». Cuadernos de Teatro Clásico, 4, pp. 95-II2.

Sarason, Seymour B. (2002): La enseñanza como arte de representación. Buenos Aires: Amorrortu.

Schechner, Richard (2002): Performance Studies. An Introduction. New York: Routledge.

Scolnicov, Hanna y Holland, Peter (eds.) (I989): The play out of context. Transferring Plays from Culture to Culture. Cambridge: Cambridge University Press.

Snell-Hornby, Mary (2006): The Turns of Translation Studies. Amsterdam: John Benjamins.

Summers, Caroline (20I6): «The playwright as epic translator? Mother Courage and the intertextual construction of an English Brecht». German Life and Letters, 62 (2), pp. 233-255.

Tacca, Óscar (1985): Las voces de la novela ( $3^{\text {a }}$ edición): Madrid: Gredos.

Törnqvist, Egil (1991): Transposing Drama. Studies in Representation. London: Macmillan. 
Veltrusky, Jirí (I990): El Drama como Literatura. Buenos Aires: Editorial Galerna.

Vieites, Manuel F. (2007): «Artes escénicas y control de calidad. Algunas cuesiones preliminars», 130 ADE/Teatro, II5, pp. I9-26.

- (2008): «El personaje dramático: aspectos generales». En J. A. Hormigón (ed.) Del personaje literario-dramático al personaje escénico. Madrid: Publicaciones de la ADE, pp. 4I-200.

- (2010): «De la teatrología: ¿Disciplina o marco disciplinar?». En: C. Rodríguez y M. F. Vieites (eds.) Teatrología. Nuevas perspectivas. Ciudad Real: Naque, pp. I3-35.

- (2015): «Teatro y traducción en Galicia. Una panorámica provisional». En: A. Luna, A. Fernández, I. Galanes y S. Montero (eds.) Literaturas extranjeras y desarrollo cultural. Berlín: Peter Lang, pp. I77-2II.

- (20I6a): "Teatro y comunicación. Un enfoque teórico». Signa, 25, pp. II53-II78.

- (20I6b): "Traducción, campo literario y sistema teatral en Galicia. Un marco teórico e histórico». Sendebar, 27, I8I-210.

- (20r6c): «La vocal que va debajo del punto o de la naturaleza posible de la dramaturgia». En: R. del Valle-Inclán, El trueno dorado (adaptación de J. A. Hormigón): Madrid: Publicaciones de la ADE, pp. $64-89$.

- (20I6d): «La traducción de obra dramática en España». En: I. Galanes, A. Luna, S. Montero y
Á. Fernández (eds.) La traducción literaria. Nuevas investigaciones. Granada: Editorial Comares, pp. 297-306.

Villegas, Juan (199I): Nueva interpretación y análisis de la obra dramática. Ottawa: Girol Books.

VV. AA. (2OI4): «El respeto para los traductores, un problema latente del teatro español». ADE/Teatro, I53, pp. I4-32.

Watzlawick, Patrick; Bavelas Janet B. y Jackson, Don D. (I99I): Teoría de la comunicación humana. Barcelona: Herder.

Yuste Frías, José (20Io): «Au seuil de la traduction: la paratraduction». En: T. Naaijkens (ed.) Event or Incident. Événement ou Incident. On the Role of Translation in the Dynamics of Cultural Exchange. $D u$ rôle des traductions dans les processus d'échanges culturels. Bern: Peter Lang, pp. 287-316.

Zatlin, Phyllis (2005): Theatrical Translation and Film Adaptation. A Practitioner's View. Clevedon: Multilingual Matters LTD.

Zhang, Baicheng (2013): «Innovative Thinking in Translation Studies: The Paradigm of Bassnett's and Lefevere's "Cultural Turn"». Theory and Practice in Language Studies, 3 (10), pp. 1919-I924.

Zuber, Ortrun (1980): The Languages of the Theatre. Problems in the Translation and Transposition of Drama. Oxford: Pergamon Press.

Zuber-Skerritt, Ortrun (ed.) (1984): Page to Stage, Theatre as translation. Amsterdam: Rodopi. 